\title{
Dynamics of fronts in optical media with linear gain and nonlinear losses
}

\author{
Izzat M. Allayarov and Eduard N. Tsoy* \\ Physical-Technical Institute of the Uzbek Academy of Sciences, \\ Bodomzor yuli st. 2-B, Tashkent, 100084, Uzbekistan \\ *Corresponding author: etsoy@uzsci.net
}

\begin{abstract}
The dynamics of fronts, or kinks, in dispersive media with gain and losses is considered. It is shown that the front parameters, such as the velocity and width, depend on initial conditions. This result is not typical for dissipative systems. For exponentially decreasing initial conditions, the relations for the front parameters are found. A presence of the global bifurcation, when a soliton solution is replaced by the front solution, is demonstrated. It is also shown that in order to observe fronts, the front velocity should be larger than the characteristic velocity of the modulational instability.
\end{abstract}




\section{INTRODUCTION}

In conservative media, light can propagate as a stable non-diverging beam due to a balance between diffraction (dispersion) and nonlinearity. Such a localized wave with a bell-shaped intensity profile in the transverse direction corresponds to a soliton, see e.g. [1, 2]. Solitons are investigated actively as important objects of nonlinear optics.

Another type of localized waves, namely, fronts (shock waves, kinks, transition waves) are studied less in optics [3-6]. A front is a local variation of the field that connects two different uniform states [7-12]. Often, one state is stable, while the other is unstable (metastable). Then, the stable state expands into the unstable one, and the front corresponds to a transition region between the two states.

The dynamics of fronts in dissipative media is a subject of extensive studies in physics [712]. An important model, that describe front propagation, is the nonlinear diffusion (ND) equation [7, 10, 11]:

$$
\partial_{z} u-D \partial_{x}^{2} u+f(u)=0
$$

where $u(x, z)$ is the real-valued field (e.g. pressure or concentration), $x$ and $z$ are the spatial and evolutional (time) coordinates, respectively, $D$ is the diffusion coefficient, and $f(u)$ is a nonlinear function. A well-known example of the ND equation is the Fisher-Kolmogorov equation [13, 14], where $f(u)=u(u-1)$. The study of this equation, started in the first half of the twentieth century, has revealed the main properties of fronts in diffusive media. Further development of the theory was connected, in particular, with the study of complexvalued fields and the combined effect of diffusion and dispersion. The corresponding model can be written as

$$
i \partial_{z} \psi+\left(\beta_{r}+i \beta_{i}\right) \partial_{x}^{2} \psi+F\left(\psi, \partial_{x} \psi, \partial_{x}^{2} \psi \ldots\right)=0
$$

where $\psi(x, z)$ is a complex field, $F$ is a function of $\psi$ and it's derivatives, and $\beta_{r}\left(\beta_{i}\right)$ characterizes dispersion (diffusion). A well studied example of model (2) is the complex Ginzburg-Landau equation that describes pattern formation in a variety of physical systems [1, 7, 9, 12].

Typically, model (2), as well as (1), possesses a family of front solutions with different spatial distributions and velocities. However, many particular examples of the model demonstrate the following property. A wide class of initial conditions evolve into a well-defined 
front with a specific velocity $v^{\star}$. This velocity depends only on the parameters of the model. In other words, the system "selects" a particular front from a variety of all possible fronts.

There are different approaches for calculation of the parameters of the selected front. The most developed approach is the theory of marginal stability (MS) that states the following [7, 8]. Let an evolutional equation in form (2) has a front solution. Then the selected front velocity $v^{\star}$ is found from the dispersion relation of the linearized Eq. (2), $\omega=\omega(k) \equiv$ $\omega_{\mathrm{r}}(k)+i \omega_{\mathrm{i}}(k)$. Namely [7, 8],

$$
v^{\star}=\omega_{\mathrm{i}}\left(k^{\star}\right) / k_{\mathrm{i}}^{\star},
$$

where the complex wavenumber $k^{\star} \equiv k_{\mathrm{r}}^{\star}+i k_{\mathrm{i}}^{\star}$ is obtained from

$$
\left.\frac{d \omega(k)}{d k}\right|_{k=k^{\star}}=\omega_{\mathrm{i}}\left(k^{\star}\right) / k_{\mathrm{i}}^{\star} .
$$

The result (3) is not proven rigorously, but it works for many types of evolutional equations.

In this paper, we analyze a limit of purely dispersive media without diffusion. This situation is typical in optics. We consider a beam propagation in media with linear gain and nonlinear losses (two-photon absorption). Such parameters are characteristic for laser systems. We demonstrate that at some conditions, the beam expansion in such media is related to the propagation of two fronts moving in opposite directions. Then, the beam evolution can be restored, to some extent, from of the front parameters. Unfortunately, the theory of MS is not useful for analysis of fronts in media with pure dispersion. We find that the front parameters in dispersive media depend not only on the the system parameters, but also on initial conditions, see Sec. III.

The optical beam dynamics in nonlinear media is described by the generalized nonlinear Schrödinger equation [1, 2]

$$
i \partial_{z} \psi+\frac{\beta}{2} \partial_{x}^{2} \psi+\left(\gamma+i \gamma_{a}\right)|\psi|^{2} \psi+i \alpha \psi=0
$$

where $\psi(x, z)$ is the envelope of the electric field, $x$ and $z$ are the transverse and longitudinal coordinates, respectively, $\beta$ is the diffraction (dispersion) coefficient, $\gamma$ is the Kerr nonlinearity parameter, $\gamma_{a}>0$ characterizes nonlinear absorption, and $\alpha<0$ is the parameter of linear gain. Equation (5) can be normalized such that $\beta=\gamma=1$, therefore we use these values in all numerical simulations.

One can distinguish two basic scenarios of the beam propagation in media with gain and losses, see Fig. 1. Let us consider, for example, a case of low initial peak intensity. Then, the 
peak intensity of the beam increases initially due to linear gain. This increase is limited by nonlinear dissipation. Intensity near the beam center keeps at a constant value due to the balance between the two effects, while intensity at the beam edges continues to rise. This process results in a formation of two fronts moving in opposite directions, see Fig. 1(a). One can see that at large $z$, fronts move with constant velocities as stationary waves.

In the second scenario, Fig. 1(b), the initial beam breaks up into several pulses that in turn generate new pulses and so on. Then, a lattice of pulses is created. That non-uniform region expands into the unstable uniform one. In fact, a lattice of pulses is formed in the first scenario as well, see Fig. 1(a). The first (second) scenario is realized when the front velocity is larger (smaller) than that of the expansion of the lattice of pulses. In this paper we are focused mainly on the first scenario, where the asymptotic dynamics of the beam is determined by the front parameters. We provide also a condition that separates the two types of the dynamics, see Sec. III.

The paper is organized as follows. The dynamical system for stationary waves is analyzed in Sec. [II. In particular, the equations for the front parameters are obtained there. Section【II discusses the condition of the front existence and comparison with numerical simulations. The results are summarized in Sec. IV.

\section{STATIONARY WAVES: FRONTS}

Fronts in Fig. 1 propagate with constant velocity, therefore in this section we study stationary wave solutions of Eq. (5). We follow the standard analysis described, for example, in Ref. [7]. We look for solutions in the following form:

$$
\psi(x, z)=a(\xi) \exp [i \phi(\xi)-i \mu z)], \quad \xi \equiv x-v z
$$

where $v$ is the wave velocity, and $\mu$ is the propagation constant. Substitution of Eq. (6) into Eq. (5) results in the dynamical system for the wave parameters:

$$
\begin{aligned}
& a^{\prime}=p a \\
& p^{\prime}=q^{2}-p^{2}-\frac{2}{\beta}\left(v q+\mu+\gamma a^{2}\right) \\
& q^{\prime}=-2 p q+\frac{2}{\beta}\left(v p-\alpha-\gamma_{a} a^{2}\right)
\end{aligned}
$$


where prime means $d / d \xi, p \equiv a^{\prime} / a$, and $q=\phi^{\prime}$. There are two types of fixed points of Eqs. (7), namely, those with vanishing amplitude ("linear" points), and those with finite amplitude ("nonlinear" points) (cf. [7]):

$$
\begin{aligned}
a_{L} & =0, \quad p_{L}=\alpha /\left(v-\beta q_{L}\right), \\
q_{L} & =\frac{1}{\beta}\left[v \pm \sqrt{b+\sqrt{b^{2}+\alpha^{2}}}\right], \\
b & \equiv v^{2} / 2+\mu \beta,
\end{aligned}
$$

and

$$
\begin{aligned}
a_{N} & =\sqrt{-\alpha / \gamma_{a}}, \quad p_{N}=0 \\
q_{N} & =\frac{1}{\beta}\left[v \pm \sqrt{v^{2}+2 \beta\left(\mu-\alpha \gamma / \gamma_{a}\right)}\right]
\end{aligned}
$$

Without loss of generality, only points with non-negative amplitude $a \geq 0$ are considered. The L-points each have one real eigenvalue and a pair of complex conjugate eigenvalues:

$$
\lambda_{1}^{(L)}=p_{L}, \quad \lambda_{2,3}^{(L)}=-2 p_{L} \pm 2 i\left(q_{L}-v / \beta\right) .
$$

The eigenvalues of each N-point are determined from the following equation

$$
\lambda^{3}+4\left(c_{N}^{2}-\frac{\alpha \gamma}{\beta \gamma_{a}}\right) \lambda-\frac{8 \alpha}{\beta} c_{N}=0, \quad c_{N}=q_{N}-v / \beta .
$$

If $c_{N}>0\left(c_{N}<0\right)$ then Eq. (11) has one negative (positive) root and a pair of complex conjugate roots with the positive (negative) real part.

A localized wave of Eq. (5) corresponds to a separatrix of dynamical system (7). In particular, a pulse is described by a separatrix that connects two L-points, while a front is described by a separatrix that connects the L- and N-points.

Equation (6) for stationary waves involves two unknown parameters, $\mu$ and $v$. In general, there are no additional conditions that fix these parameters. The theory of MS is developed for dissipative systems with both dispersion and diffusion, i.e. when parameter $\beta$ is complex. For purely dispersive media, when $\beta$ in Eq. (5) is real, the theory of MS gives that any value of $v$ is possible.

For Eq. (5), we find a result which is not typical for dissipative systems. From our analysis, we conclude that the front velocity is defined also by initial conditions. This is in contrast to dissipative diffusive media, where $v$ depends mainly on the system parameters. 
There are no regular methods to obtain the front parameters for arbitrary initial conditions. Let us consider a particular initial condition $\psi(x, 0) \equiv \psi_{0}(x)=a_{0}(x) \exp \left[i \phi_{0}(x)\right]$ with the following asymptotic behavior for amplitude $a_{0}(x)$ and phase $\phi_{0}(x)$ :

$$
a_{0}(x) \sim e^{-|x| / w_{0}}, \quad \phi_{0}^{\prime}(x) \sim q_{0} \text { at }|x| \rightarrow \infty .
$$

We find that a front developed from such initial conditions moves uniformly (see Fig. 1(a)), while $v$ and $\mu$ are defined from Eq. (8) with substitution

$$
p_{L}=\mp 1 / w_{0}, \quad q_{L}=q_{0},
$$

or explicitly

$$
\begin{aligned}
v & =\mp \alpha w_{0}+\beta q_{0}, \\
\mu & = \pm \alpha w_{0} q_{0}-\frac{\beta}{2}\left(q_{0}^{2}+1 / w_{0}^{2}\right) .
\end{aligned}
$$

If $a=0$ is at $x=+\infty(x=-\infty)$ then the upper (lower) sign in Eqs. (13) and (14) should be chosen. Therefore, we come to an interesting result that the parameters of a stationary front are governed by the parameters of the L-point only, or, in other words, by the parameters of small-amplitude waves of Eq. (5).

In the equation for $v$, the second term is the phase velocity of linear waves. Then, the first term is the relative velocity of the front in the corresponding reference frame. We use this relative velocity in Sec. III.

Figure 2 shows good agreement between the values of the front velocity found from numerical simulation of Eq. (5) and calculated using Eqs. (14). The initial condition is taken as

$$
\psi_{0}(x)=a_{N} \operatorname{sech}\left(x / w_{0}\right) .
$$

The velocity is determined numerically for large $z$, when all transient processes are ended. All points in Fig. 2 corresponds to the first scenario, when well-pronounced fronts are developed, see Fig. 1(a). This scenario is realized for sufficiently large $w_{0}$. This is discussed in detail in Sec. III.

We extend our results further to beams with asymptotic behavior different from that in Eq. (12). Let us consider for simplicity the real $\psi_{0}(x)$. If $\psi_{0}(x)$ decreases at $|x| \rightarrow \infty$ slower (faster) than exponentially, then the emerging fronts move decelerating (accelerating). Figure 3 represents such dynamics for a Gaussian pulse and a Lorentzian pulse, respectively. 
We obtain that even in these non-stationary cases, Eqs. (8) and (13) can still be used for estimation of front parameters, provided that $w_{0}$ and $q_{0}$ are corresponding function of $z$.

The analysis above shows that media with linear amplification and nonlinear dissipation can be used to distinguish beams with different asymptotics. A deviation of the beam's asymptotic behavior from an exponential one results in a deviation of the dependence of the beam width on $z$ from a straight line.

\section{EXISTENCE OF FRONTS}

There are two questions regarding the front existence. The first question is for what values of the system parameters does the L-N separatrix exist. The second question is what are the conditions for development of well-pronounced fronts. We discuss these question in the present section.

There are no general methods to determine the existence of L-N separatrices. However, our study uncover a notable property of system (7). It is known [15] that Eq. (5) has an exact soliton solution. It corresponds to a L-L separatrix of Eqs. (7). On the other hand, numerical simulations of Eq. (5) show unambiguously the existence of fronts, or L-N separatrices, for other sets of the system parameters. Also, analysis of the eigenvalues of the L-points reveals that only a single trajectory goes out of plane $a=0$ from the L-point. It means that the L-L and L-N separatrices cannot co-exist for a given set of the parameters. These facts indicate a presence of a global bifurcation [16] in system (7), and therefore in Eq. (5). Namely, depending on the parameters, the separatrix that starts from the L-point, ends at either the another L-point or the N-point as $\xi \rightarrow \pm \infty$, depending on the parameters. At the same time, the local properties of model (7), in particular, the types of the fixed points, do not change near the bifurcation.

Let us fix all the parameters in Eqs. (77), except of $\mu$. We find that the L-L (L-N) separatrix exists below (above) a bifurcation value $\mu_{B}$. Figure 4 shows separatrices that go from (to) the L- and N-points for different sets of the parameters. Only trajectories with real eigenvalues, $\lambda_{L}$ and $\lambda_{N}$, are shown. In order to reconstruct the heteroclinic trajectories in Fig. 4. we take an initial condition at the vicinity of the fixed point along the direction of the corresponding eigenvector. Each trajectory is checked by integrating backward on $\xi$ from the end point. 
In Fig. 4(a), $\mu=-0.7104<\mu_{B}$, there is a separatrix that connects the two L-points. One separatrix of the N-point is located in a region $a>1$. The other separatrix of the N-point goes to (from) a region $a<1$, intertwining with the separatrix of the second N-point. In Fig. $4(\mathrm{~b}), \mu=0.85>\mu_{B}$, there is a separatrix that connect points of distinct types.

The value $\mu=-0.7104$ for $v=0, \alpha=-0.5$ and $\gamma_{a}=0.5$ corresponds to the exact soliton solution [15]. The value $\mu=0.85 \equiv \bar{\mu}_{B}(v=0)$ for the same values of the other parameters can be considered as an upper estimate of $\mu_{B}$. We find $\bar{\mu}_{B}(0)$ such that integrating forward or backward on $\xi$ from the one of the fixed point, the trajectory approaches the other fixed point closer than 0.002 in absolute units. For other values of $v$, the bifurcation value $\mu_{B}$ (or $\bar{\mu}_{B}$ ) can be calculated using the Galilean invariance, $\mu_{B}(v)=\mu_{B}(0)-v^{2} / 2$. We also find that $\bar{\mu}_{B}$ depends weakly on $\gamma_{a}$ and almost linearly on $\alpha$.

Now we turn to the question of the front development. First, we analyze real initial conditions, i.e. without phase modulations. We find that the first scenario with well-defined fronts is realized, when the front velocity is larger than the threshold velocity $v_{t h}$. The threshold $v_{t h}$ is the velocity of an expansion of the pulse region. The later, we believe, is related to the modulational instability of the plane wave with amplitude $a_{N}$. Namely, a tail of a pulse plays a role of a perturbation of the uniform (L- or N-) state. Due to instability, new pulses are generated from this perturbation resulting in the expansion of the pulse region.

In order to estimate $v_{t h}$, we perform the analysis of the modulational instability of Eq. (55). Let us consider the plane-wave solution of Eq. (5):

$$
\psi(x, z)=a_{N} e^{i \varphi(x)}, \quad \varphi(x)=(k x-\omega z),
$$

where

$$
\omega=\beta k^{2} / 2+\alpha \gamma / \gamma_{a} .
$$

Equation for small modulations $u(x, z)$ can be obtained by substitution of the field in the form

$$
\psi=\left[a_{N}+u(x, z)\right] e^{i \varphi(x)}
$$

into Eq. (5) and linearizing on $u$. Then, assuming $u \sim \exp [i(K x-\Omega z)]$, one can derive the dispersion relation of modulations:

$$
\Omega=\beta k K+i \alpha \pm\left[-\alpha^{2}+\frac{\beta K^{2}}{2}\left(\frac{\beta K^{2}}{2}+2 \frac{\alpha \gamma}{\gamma_{a}}\right)\right]^{1 / 2} .
$$


It follows from the analysis of Eq. (19) that the plane wave (16) is unstable when $|K|<$ $2\left[-\alpha \gamma /\left(\beta \gamma_{a}\right)\right]^{1 / 2}$. The instability gain, $g(K) \equiv \operatorname{Im}[\Omega(K)]$, has a maximum at $K=K_{m}=$ $\left[-2 \alpha \gamma /\left(\beta \gamma_{a}\right)\right]^{1 / 2}$. Then the characteristic velocity $v_{M I}$ of the instability expansion is estimated as a ratio of characteristic scales on $z$ and $x$, namely the maximum of instability gain and the corresponding wavenumber:

$$
v_{M I} \equiv \frac{g\left(K_{m}\right)}{K_{m}}
$$

Numerical simulations of Eq. (5) reveal that the threshold velocity can be determined as $v_{t h}=C v_{M I}$. Fitting of this equation with numerical data gives $C \approx 4.7$ for $\beta=\gamma=1$. Then, well-defined fronts exist if

$$
\left|\alpha w_{0}\right|>v_{t h} \equiv C \sqrt{\frac{|\alpha| \beta \gamma_{a}}{2 \gamma}}\left(\sqrt{1+\gamma^{2} / \gamma_{a}^{2}}-1\right) .
$$

This condition defines the parameter $w_{0}$ of the initial profile that develops into two fronts.

The dependence of $v_{t h}$ on the system parameters found numerically and using Eq. (21) is shown in Fig. 5, For a given set of the system parameters, we change the initial width $w_{0}$, until the velocity of the emerging fronts exceeds the velocity of pulse spreading. The threshold is not sharp, therefore the relative error of $v_{t h}$ is in order 0.1. Nevertheless, we see a good agreement between numerical results and Eq. (21). We also calculate numerically the propagation constant $\mu_{t h}$ of the front at $v=v_{t h}$. Taking $\mu$ as a control parameter, we find that the formation of well-defined fronts occurs at larger $\mu$ than the global bifurcation, $\mu_{t h}>\bar{\mu}_{B}\left(v_{t h}\right)$.

An addition of the linear phase to initial conditions results in a constant shift of the whole beam profile with the corresponding phase velocity. This is a manifestation of the Galilean invariance discussed in Sec. II. In this case one should compare the relative front velocity and $v_{t h}$, obtaining the same Eq. (21). Therefore, Eq. (21) is valid also for initial conditions with linear modulation of the phase.

\section{CONCLUSION}

We have demonstrated that in dispersive media with gain and losses, a beam propagates for some set of the system parameters in a form of two fronts moving in opposite directions. It has been shown that the front parameters depend on the system parameters, as well as on 
initial conditions. The presence of global bifurcation of stationary waves in Eqs. (17) is found. The threshold for development of fronts has been obtained. A possibility to use dispersive media to distinguish beams with different asymptotic behavior has been suggested.

[1] N. N. Akhmediev and A. Ankiewicz, Solitons, Nonlinear Pulses and Beams, Chapman and Hall, London, 1997.

[2] Yu. S. Kivshar and G. Agrawal, Optical Solitons: From Fibers to Photonic Crystals, Academic Press, 2003.

[3] G. P. Agrawal and C. Headley, Kink solitons and optical shocks in dispersive nonlinear media, Phys. Rev. A 46 (1992) 1573.

[4] S. Darmanyan, A. Kamchatnov, and F. Lederer, Optical shock waves in media with quadratic nonlinearity, Phys. Rev. E 58 (1998) R4120.

[5] M. I. Carvalho, A. G. Grandpierre, D. N. Christodoulides, and M. Segev, Optical spatial shock waves in photorefractive media, Phys. Rev. E 62 (2000) 8657.

[6] B. Freedman, T. Carmon, M. I. Carvalho, M. Segev, and D. N. Christodoulides, Observation of locked optical kink-antikink spatial shock waves, Phys. Rev. Lett. 91 (2003) 133902.

[7] W. van Saarloos and P. C. Hohenberg, Fronts, pulses, sources and sinks in generalized complex Ginzburg-Landau equations, Physica D 56 (1992) 303.

[8] W. van Saarloos, Front propagation into unstable states, Phys. Rep. 386 (2003) 29.

[9] I. S. Aranson and L. Kramer, The world of the complex Ginzburg-Landau equation, Rev. Mod. Phys., 74 (2002) 99.

[10] V. A. Vasiliev, Yu. M. Romanovsky, and V. G. Yakhno, Autowave Processes, Nauka, Moscow, 1987 (in Russian).

[11] A. I. Volpert, Vit. A. Volpert, Vl. A. Volpert, Traveling Wave Solutions of Parabolic Systems, Translation of Math. Monograph, v. 140, Am. Math. Soc., 2000.

[12] N. Akhmediev A. Ankiewicz (Eds.), Dissipative Solitons, Lect. Notes Phys. v.661, Springer, Berlin, 2005.

[13] R. H. Fisher, The wave of advance of advantageous genes, Ann. Eugen. 7 (1937) 355.

[14] A. Kolmogorov, I. Petrovsky and N. Piskunov, Étude de l'équation de la chaleur avec croissance de la quantité de matière et son application à un problème biologique, Bull. Univ. 
Moscow Ser. Int. A 1 (1937) 1.

[15] K. Nozaki and N. Bekki, Exact solutions of the generalized Ginzburg-Landau equation, J. Phys. Soc. Japan, 53 (1984) 1581-1582.

[16] J. Guckenheimer and P. Holmes, Nonlinear Oscillations, Dynamical Systems, and Bifurcations of Vector Fields, Appl. Math. Sci, v. 42, Springer, 1983. 

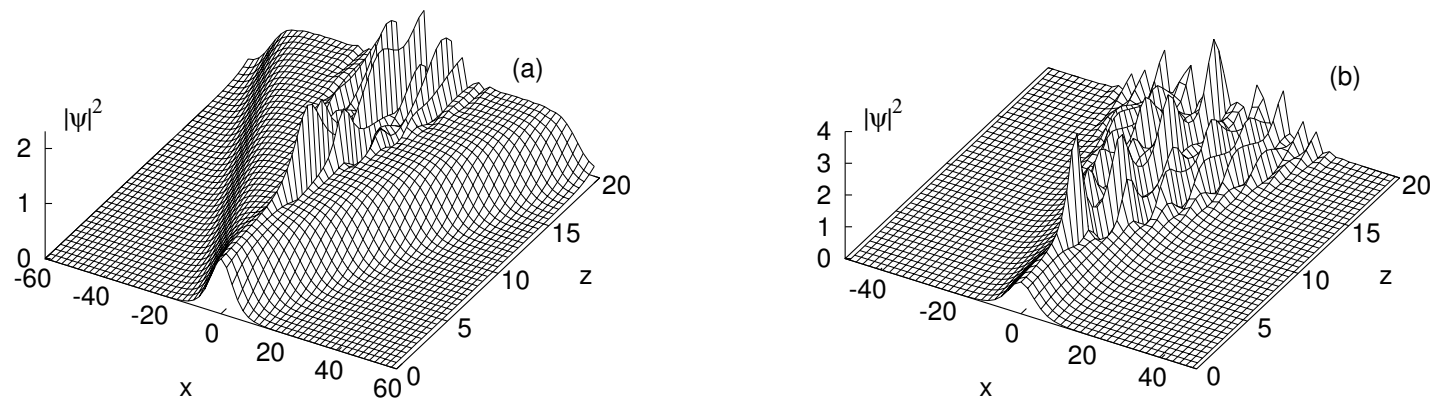

FIG. 1. The beam dynamics described by Eq. (5) for initial condition $\psi(x, 0)=\operatorname{sech}\left(x / w_{0}\right)$. Plots show the evolution of the beam intensity $|\psi|^{2}$. The parameters are (a) $\alpha=-0.5, \gamma_{a}=0.5$ and $w_{0}=5$, (b) $\alpha=-0.2, \gamma_{a}=0.2$ and $w_{0}=5$.

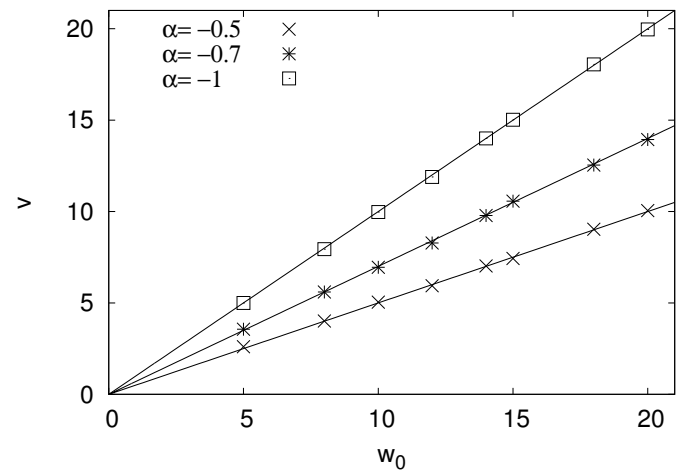

FIG. 2. The dependence of the front velocity on $w_{0}$ for $\gamma_{a}=0.5$ and different values of $\alpha$. Points correspond to result of numerical simulations of Eq. (15). Lines are found from Eqs. (14). 

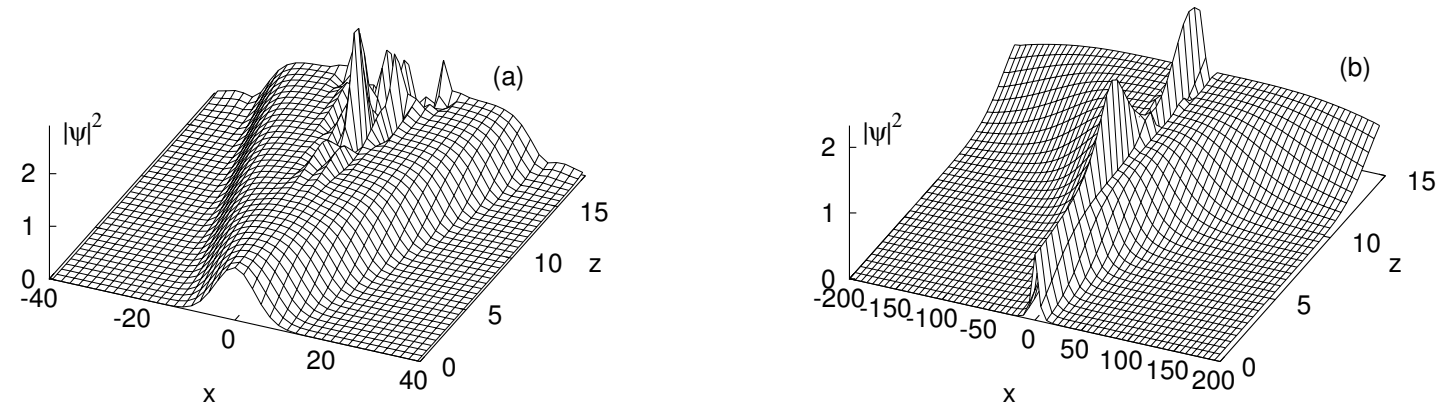

FIG. 3. The dynamics of (a) a Gaussian pulse, $\psi(x, 0)=\exp \left[-(x / 6)^{2} / 2\right]$, and (b) a Lorentzian pulse, $\psi(x, 0)=\left[(x / 6)^{2}+1\right]^{-1}$. The parameters are $\alpha=-0.5$ and $\gamma_{a}=0.5$. 

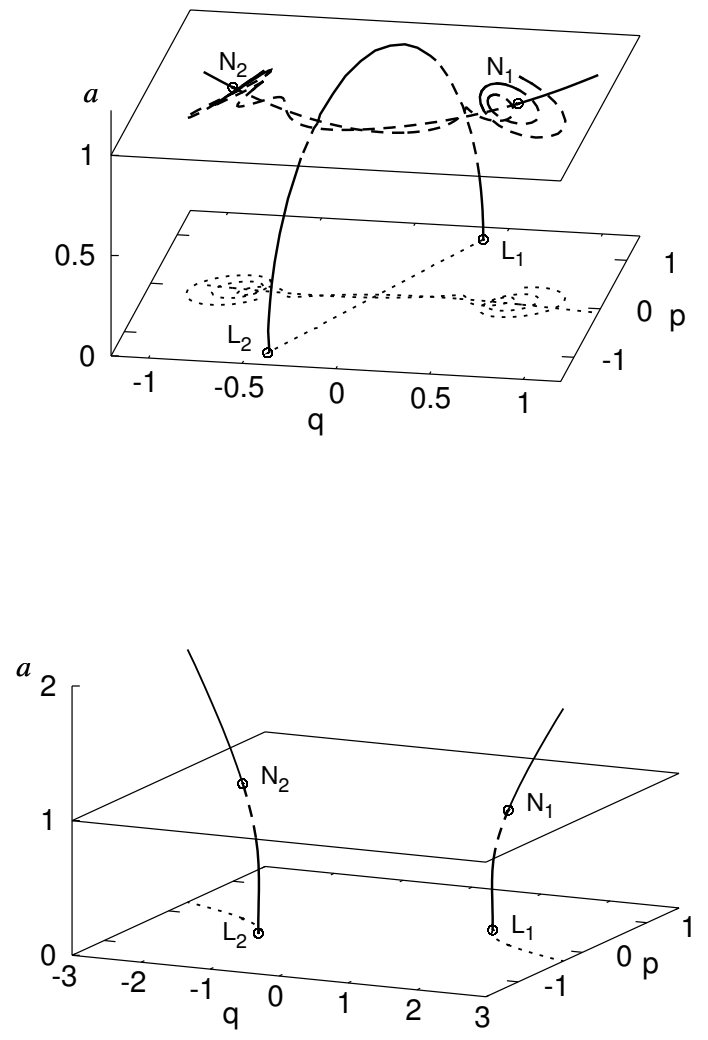

FIG. 4. Separatrices in the phase space of Eqs. (7) for $v=0, \alpha=-0.5$, and $\gamma_{a}=0.5$. The L-points (N-points) are labelled as $L_{1}$ and $L_{2}\left(N_{1}\right.$ and $\left.N_{2}\right)$. (a) The L-points are connected via the L-L separatrix at $\mu=-0.7104$. (b) There are two L-N separatrices at $\mu=0.85$. The dotted lines show the projections of the separatrices on plane $a=0$. 


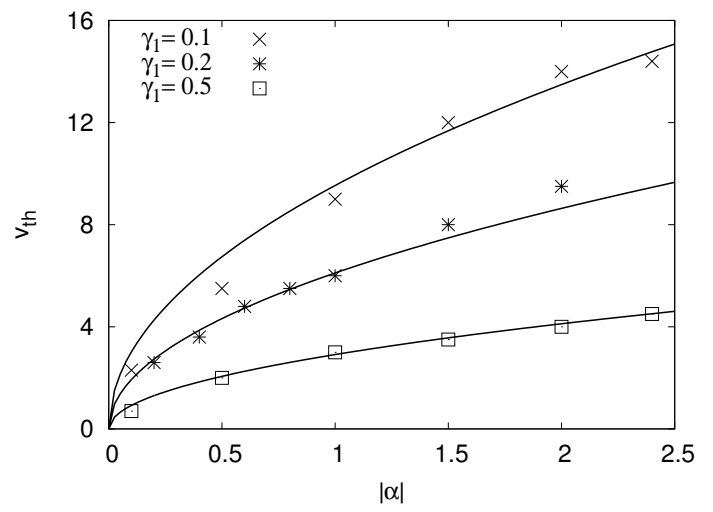

FIG. 5. The threshold velocity as a function of $|\alpha|$ for different $\gamma_{a}$. Points correspond to numerical simulations of Eq. (5). Lines correspond to Eq. (21), $C \approx 4.7$. 